\title{
Self-consistent optical potential for $\alpha$-particles at low energies
}

\author{
M. Avrigeanu ${ }^{1, a}$, W. von Oertzen ${ }^{2}$, A. Obreja ${ }^{1}$, F.L. Roman ${ }^{1}$, and V. Avrigeanu ${ }^{1}$ \\ 1 "Horia Hulubei" National Institute for Physics and Nuclear Engineering, P.O. Box MG-6, 76900 Bucharest, Romania \\ 2 Freie Universität Berlin, Fachbereich Physik, 14195 Berlin, and Hahn-Meitner-Institut, 14109 Berlin, Germany
}

\begin{abstract}
A previous semi-microscopic analysis based on the double folding model, for the $\alpha$-particle elastic scattering on $A \sim 100$ nuclei at energies below $32 \mathrm{MeV}$, is enlarged for medium mass nuclei up to $A \sim 120$ and energies below $50 \mathrm{MeV}$. The energy-dependent phenomenological imaginary part for this semi-microscopic optical model potential (OMP) was obtained, making use also of the dispersive correction to the microscopic real potential. A regional OMP parameter set for low-energy $\alpha$-particles has been obtained as well for nuclei within the abovementioned mass range. It has been proved able to describe both scattering and $\alpha$-particle induced reaction data, through the statistical model analysis of most accurate data of total $\alpha$-reaction cross sections for ${ }^{48} \mathrm{Ti}$ and ${ }^{51} \mathrm{~V}$, and $(\alpha, \mathrm{n})$ reaction cross sections for the target nuclei ${ }^{45} \mathrm{Sc},{ }^{46,48} \mathrm{Ti},{ }^{51} \mathrm{~V},{ }^{50} \mathrm{Cr}$ and ${ }^{54} \mathrm{Fe}$ and incident energies lower than $11-12 \mathrm{MeV}$.
\end{abstract}

\section{Introduction}

The optical model potential (OMP) analyses for $\alpha$-particles at low energies pointed out two main questions which are still open (e.g., [1] and refs. therein), namely (i) the OMP parameter sets obtained from $\alpha$-particle elastic scattering at high energies $(>80 \mathrm{MeV})[2]$ do not describe either the low-energy $(<40 \mathrm{MeV})$ elastic scattering or the complete fusion data, and (ii) the statistical $\alpha$-particle emission is underestimated by the OMPs that account for elastic scattering on the (cold) ground-state nuclei [3]. In the latter case, the need for new physics in potentials to describe nuclear de-excitation within the statistical model calculations was earlier pointed out [4] and effects due to changes of the nuclear density at a finite temperature have been considered in this respect within the double folding model (DFM) of the alpha-nucleus real part of the optical potential [5]. At the same time, the high precision of recent measurements of $\alpha$-particle elastic-scattering data, e.g., [6-8], makes possible to point out additional features of the $\alpha$-particles scattering at low energy and, consequently, further limits and possible improvement of global OMP parameters obtained previously through semi-microscopic analysis of the low-energy $\alpha$-particle elastic scattering [9].

In order to avoid the questions arising due to the remaining parameters needed in the pre-equilibrium emission and statistical models, the available experimental $\alpha$-induced or $(\mathrm{n}, \alpha)$ reaction cross sections were not taken into account in the previous analyses of refs. [1,9]. Thus significant uncertainties of the calculated reaction cross sections [10] have had no effect on the optical potential setting up while a useful completion of this work should be the subsequent application of the related OMP parameter set to the analysis of the available reaction cross sections. The ultimate awareness in this respect should be the above-mentioned distinction between either the incoming or outgoing reaction channels within which the $\alpha$-particles are concerned. Consequently, the OMP parameter sets established by analysis of the elastic scattering should be involved in the survey of $\alpha$-particle

\footnotetext{
${ }^{a}$ Presenting author, e-mail: mavrig@ifin.nipne.ro
}

Table 1. The experimental data of $\alpha$-particle elastic-scattering analysed in this work.

\begin{tabular}{cc}
\hline Target & $E_{d}(\mathrm{MeV})$ \\
\hline${ }^{50} \mathrm{Ti}$ & $25,27.1,29.1,31,33,35.1,37.1$, \\
${ }^{51} \mathrm{~V},{ }^{50,52,53} \mathrm{Cr},{ }^{56,58} \mathrm{Fe}$, & 25 \\
${ }^{59} \mathrm{Co},{ }^{63} \mathrm{Cu}$ & $12.8,14.56,16.34,18.13$ \\
${ }^{50} \mathrm{Cr},{ }^{62} \mathrm{Ni}$ & $23,25,27.1,29.1,31,33,35.1,37.1$, \\
${ }^{52} \mathrm{Cr}$ & $39,41.1,43,45,46.8$ \\
& 26.5 \\
${ }^{56} \mathrm{Fe}$ & $18,21,24.1,25,27,32.3$ \\
${ }^{58} \mathrm{Ni}$ & 29,34 \\
${ }^{58,60,62,64} \mathrm{Ni}$ & $21,23.4,25$ \\
${ }^{60} \mathrm{Ni}$ & 15 \\
${ }^{89} \mathrm{Y},{ }^{90,91} \mathrm{Zr}$ & $13.8,16.4,19.5$ \\
${ }^{90} \mathrm{Zr},{ }^{107} \mathrm{Ag}$ & 25.2 \\
${ }^{92} \mathrm{Mo}$ & $16.2,19.4$ \\
${ }^{94} \mathrm{Mo},{ }^{107} \mathrm{Ag},{ }^{116,122,124} \mathrm{Sn}$ & 14.4 \\
${ }^{89} \mathrm{Y}$ & 19.5 \\
${ }^{112} \mathrm{Sn}$ & \\
${ }^{112,124} \mathrm{Sn}$ & \\
\hline
\end{tabular}

induced reaction data including the total $\alpha$-reaction cross sections [11]. All available data of this sort for nuclei with $A \sim 50$ have thus been used in the present work (table 1), while the less proper use [12] in the same cases of the phenomenological $\alpha$-particle OMPs established for emission from excited nuclei [3] has been again underlined. Actually, for calculation of the reaction cross section we have used for the rest of model quantities a consistent parameter set established within recent analyses of the available fast-neutron activation cross sections for all stable isotopes of $\mathrm{V}, \mathrm{Mn}, \mathrm{Co}$, $\mathrm{Ni}$ and $\mathrm{Cu}$ [13-15], adopted through an analysis of various independent experimental data. For this only reason, it becomes possible to focus on the uncertainties of the OMP parameters and look for their improvement and understanding on the basis of microscopic models. 

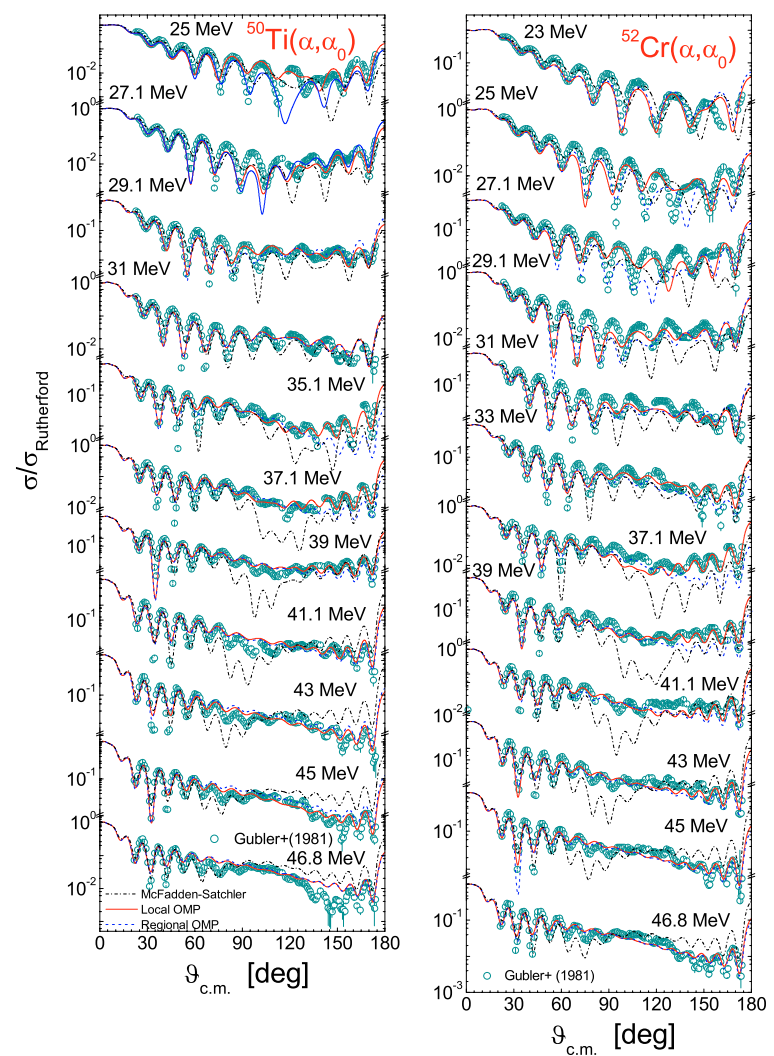

Fig. 1. Comparison of the experimental [18] and calculated angular distributions of $\alpha$-particle elastic scattering on ${ }^{50} \mathrm{Ti}$ and ${ }^{52} \mathrm{Cr}$ between 23 and $47 \mathrm{MeV}$, using the phenomenological OMP local (solid curves) and regional (dashed curves) parameters as well as the global OMP parameter set of McFadden and Satchler [19] (dashdotted curves).

\section{Semi-microscopic and phenomenological optical potentials}

Similarly to the semi-microscopic analysis [1] of $\alpha$-particle elastic scattering on $A \sim 100$ nuclei, at energies below $32 \mathrm{MeV}$, we have obtained the energy-dependent phenomenological imaginary potential making use also of the corresponding dispersive correction to the microscopic "parameter free" DF real potential. Then a full phenomenological analysis of the same data has been carried out with the imaginary part unchanged from the former semi-microscopic analysis, except minor adjustment of the potential depths. The computer code SCAT2 [16] has been used in this respect, modified to include the semi-microscopic DF potential of ref. [17] as an option for the OMP real part. The advantage of having already well settled about half of the usual OMP parameters increases obviously the accuracy of the local fit of data (figs. 1-6).

Unfortunately a $\chi^{2}$ analysis, which would have been the optimal procedure, has not been possible in all cases due to the lack of numerical cross-sections including the corresponding errors for some of the experimental data given in table 1 . Nevertheless it was applied for the more recent data, using the original errors, while the good overall agreement finally obtained for various target nuclei can be considered as a suitable validation of the actual OMP parameter sets.
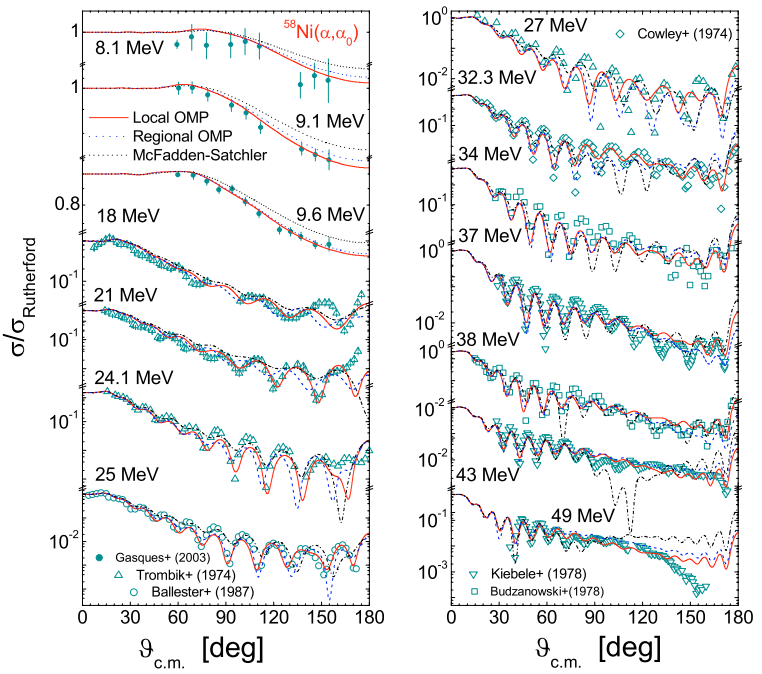

Fig. 2. Same as figure 1 but for $\alpha$-particles scattered on ${ }^{58} \mathrm{Ni}$ between 8 and $49 \mathrm{MeV}$.
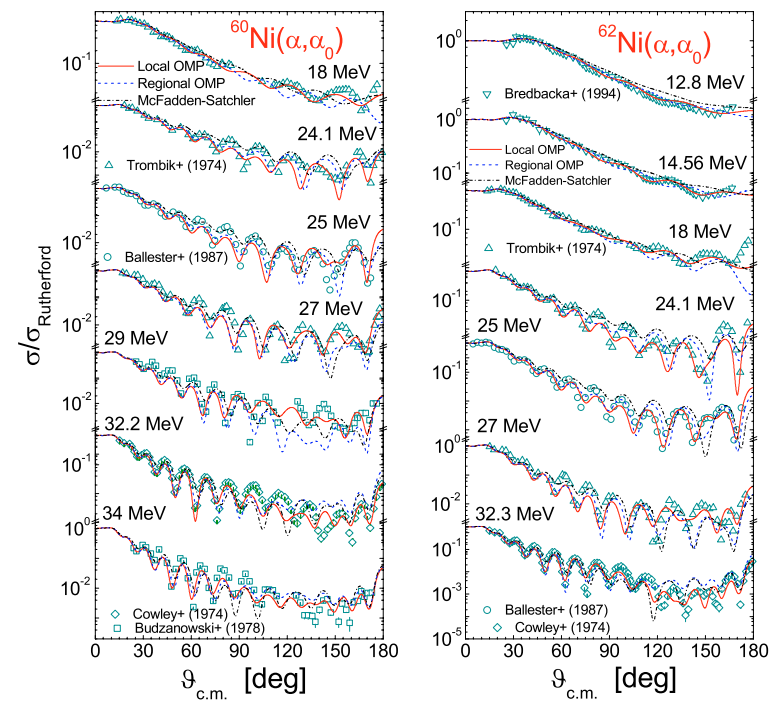

Fig. 3. Same as figure 1 but for $\alpha$-particles scattered on ${ }^{60,62} \mathrm{Ni}$ between 12.8 and $34 \mathrm{MeV}$.

Average mass-, charge-, and energy dependences of the local OMP parameters, with forms rather similar to those introduced by Nolte et al. [2] above $80 \mathrm{MeV}$, have been obtained in the end of the latter step of our analysis, leading to a regional optical potential (ROP) with Woods-Saxon (WS) form factors for the real and imaginary parts. The angular distributions calculated using these average parameters as well as the global parameter set of McFadden and Satchler [19] are also shown in figures $1-6$, in order to establish the usefulness of the former potential and the deviations of its predictions from the local results analysis. On the whole, we find that a rather suitable description of the analyzed data is provided by OMPs with real-potential diffuseness $a_{R} \sim 0.61 \mathrm{fm}[1,19]$ noticeably lower than $a_{R}$ values which are needed in order to describe $\alpha$-particle emission from excited compound nuclei [3,5].

For the general discussion and for comparison with other work, we have also calculated the volume integrals per interacting nucleon pair of the real $\left(J_{R}\right)$ and imaginary volume 

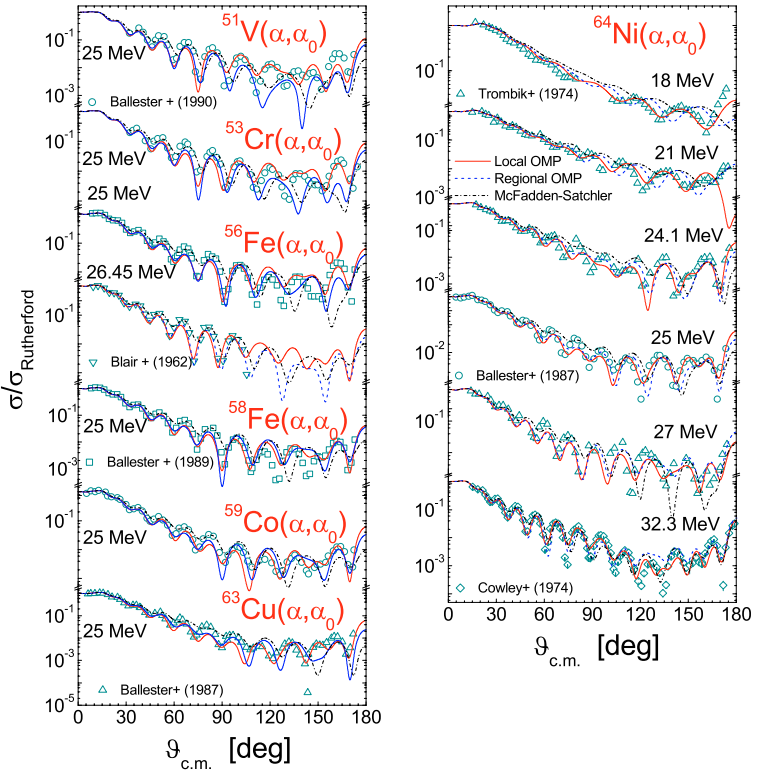

Fig. 4. Same as figure 1 but for $\alpha$-particles scattered on ${ }^{51} \mathrm{~V},{ }^{53} \mathrm{Cr}$, ${ }^{56,58} \mathrm{Fe},{ }^{59} \mathrm{Co},{ }^{63} \mathrm{Cu}$ and ${ }^{64} \mathrm{Ni}$ between 18 and $32 \mathrm{MeV}$.
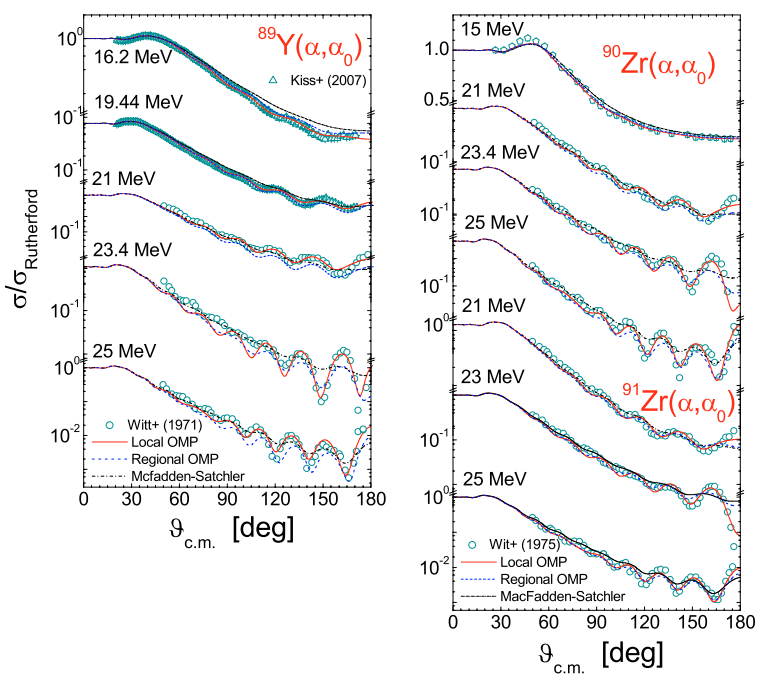

Fig. 5. Same as figure 1 but for $\alpha$-particles scattered on ${ }^{89} \mathrm{Y}$ and ${ }^{90,91} \mathrm{Zr}$ between 15 and $25 \mathrm{MeV}$.

and surface components of the present phenomenological potential. Thus, we found $J_{R}$ values which are increased by $\sim 25 \%$ with respect to those obtained previously [1]. At the same time, they are lower by a similar amount with respect to the corresponding values provided by the well-known global parameter set of McFadden and Satchler [19]. These differences come mainly from the different radial dependence of the corresponding potentials (e.g., fig. 10 of ref. [1]), while the rather similar good description of the elastic scattering data at low energies is explained by the fact that these potentials are almost identical in the tail region.

\section{$3(\alpha, \mathrm{n})$ and total $\alpha$-reaction cross section analysis}

A key assessment of the ROP obtained by the semimicroscopic and phenomenological analyses has consisted
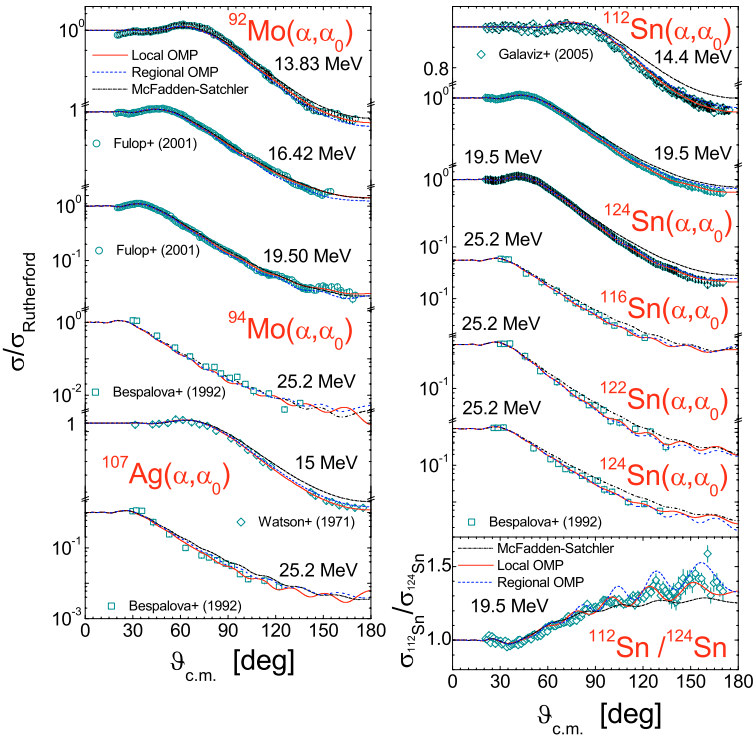

Fig. 6. Same as figure 1 but for $\alpha$-particles scattered on ${ }^{92} \mathrm{Mo},{ }^{107} \mathrm{Ag}$, and ${ }^{112,116,122,124} \mathrm{Sn}$ between 13.8 and $25.2 \mathrm{MeV}$, and (bottom-right) the corresponding ratio of the elastic scattering cross sections of ${ }^{112} \mathrm{Sn}$ and ${ }^{124} \mathrm{Sn}$ at $E_{\alpha}=19.5 \mathrm{MeV}$.

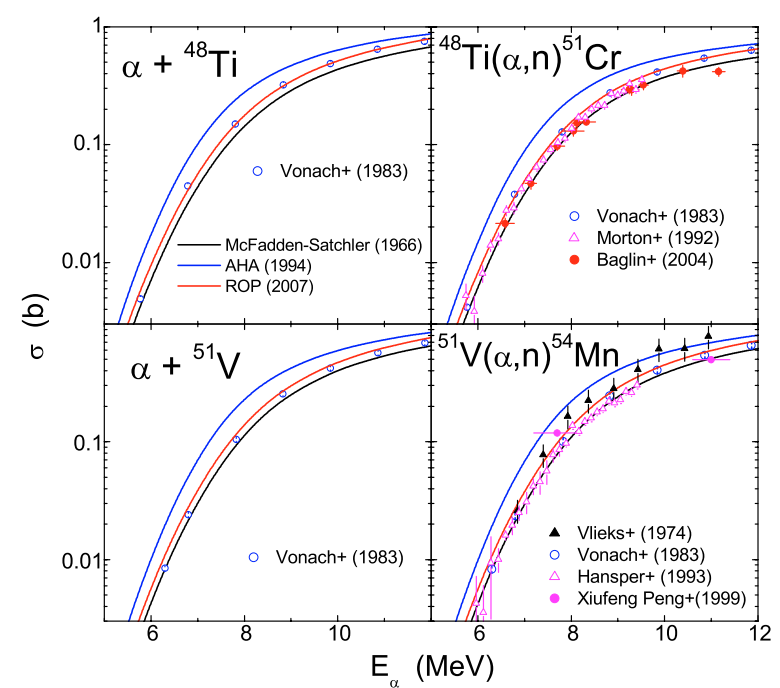

Fig. 7. Comparison of measured [11] and calculated $(\alpha, \mathrm{n})$ and total $\alpha$-reaction cross sections for ${ }^{48} \mathrm{Ti}$ and ${ }^{51} \mathrm{~V}$ using present ROP (solid) and OMPs of refs. [3,19] (dotted and dashed curves, respectively).

in the analysis of the particularly accurate $(\alpha, \mathrm{n})$ and total $\alpha$-reaction cross sections for ${ }^{48} \mathrm{Ti}$ and ${ }^{51} \mathrm{~V}$ measured and respectively established by Vonach et al. [11]. Their obvious overestimation by the OMP found able to describe the $\alpha$-particle emission was pointed out from the beginning [3], being less apparent its use within actual analyses of $\alpha$-particle elastic scattering [12]. The importance of an enlarged consideration on accurate description of $\alpha$-particle induced reactions by the actual ROP led us to include in this work the analysis of all $(\alpha, \mathrm{n})$ reaction cross sections available within the mass range $A \sim 50$ below the incident energy of $10-12 \mathrm{MeV}$. The focus on this energy range, most important for fields like nuclear astrophysics, is due to the largest sensitivity of the calculated cross sections to the $\alpha$-particle 


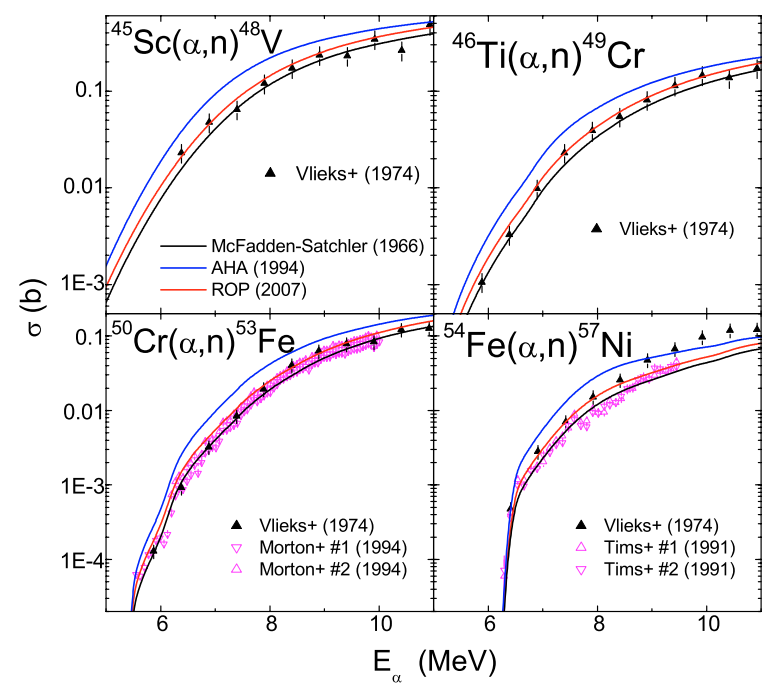

Fig. 8. Same as figure 1 but for $(\alpha, \mathrm{n})$ reaction cross sections for ${ }^{45} \mathrm{Sc}$, ${ }^{46} \mathrm{Ti},{ }^{50} \mathrm{Cr}$, and ${ }^{54} \mathrm{Fe}$. The experimental data are from ref. [18].

OMP while the rest of the statistical approach parameters are rather meaningless. On the other hand, the only few measurements of the $(\alpha, \alpha)$ data performed at these energies underline the usefulness of complementary studies provided that uncertainties of other model items could be avoided.

The Hauser-Feshbach statistical model calculations of these $(\alpha, \mathrm{n})$ reaction cross sections have been carried out similarly to the previous analysis of (n, $\alpha$ ) reaction cross section for $A \sim 90$ [5] except different optical potentials used in the incident and emergent $\alpha$-particle channels. A consistent local parameter set for the mass range $A \sim 60$ was used, adopted recently [13-15] through an analysis of various independent data. Furthermore, these parameters were involved within an updated version of the computer code STAPRE-H95 [20] for the description of the whole set of available experimental neutron activation data for all stable isotopes of $\mathrm{V}, \mathrm{Mn}, \mathrm{Co}, \mathrm{Ni}$ and $\mathrm{Cu}$. The reaction cross sections calculated by means of the same OMP parameters obtained throughout the analysis of the $(\alpha, \alpha)$ angular distributions (figs. 1-6) are shown in figures 7-8. Finally it results a description of both scattering and $\alpha$-induced reaction data by the present ROP which is well improved compared to the global parameter sets of McFadden and Satchler [19].

Further work should be devoted to semi-microscopic description of $\alpha$-particle emission for $A \sim 60$ in order to provide additional evidence for the temperature dependence of the nuclear density distribution function which could be the missing degree of freedom that needs to be included in conventional statistical model calculations even for nuclear temperature smaller than $2 \mathrm{MeV}$ [5].
Special thanks go to Prof. Zsolt Fülöp for providing the results of ref. [8] in tabular form prior to publication. This work was supported in part by the Contract No. ERB-5005-CT-990101 of EURATOMMEdC (Bucharest) Fusion Association, the Research Contract No. 12422 of the International Atomic Energy Agency, and MEdC Contract No. CEEX-05-D10-48.

\section{References}

1. M. Avrigeanu, W. von Oertzen, A.J.M. Plompen, V. Avrigeanu, Nucl. Phys. A 723, 104 (2003).

2. M. Nolte, H. Machner, J. Bojowals, Phys. Rev. C 36, 1312 (1987).

3. V. Avrigeanu, P.E. Hodgson, M. Avrigeanu, Phys. Rev. C 49, 2136 (1994).

4. G. La Rana et al., Phys. Rev. C 35, 373 (1987); G. D.J. Moses et al., Phys. Rev. C 36, 422 (1987); R. Lacey et al., Phys. Lett. B 191, 253 (1987); G. Batko, O. Civitarese, Phys. Rev. C 37, 2647 (1988); G. Bozzolo et al., Phys. Lett. B 219, 161 (1989).

5. M. Avrigeanu, W. von Oertzen, V. Avrigeanu, Nucl. Phys. A 764, 246 (2006).

6. Zs. Fülöp, Gy. Gyürky, Z. Máté, E. Somorjai, L. Zolnai, D. Galaviz, M. Babilon, P. Mohr, A. Zilges, T. Rauscher, H. Oberhummer, G. Staudt, Phys. Rev. C 64, 065805 (2001).

7. D. Galaviz, Zs. Fülöp et al., Phys. Rev. C 71, 065802 (2005).

8. G.G. Kiss, Zs. Fülöp, Gy. Gyrky, Z. Mt, E. Samorjai, D. Galaviz, S. Müller, A. Zilges, P. Mohr, M. Avrigeanu, in Proc. of Europhysics Conf. on Nuclear Physics in Astrophysics III, Dresden, March 26-31, 2007 (in press).

9. M. Avrigeanu, V. Avrigeanu, Phys. Rev. C 73, 038801 (2006).

10. P. Demetriou, C. Grama, S. Goriely, Nucl. Phys. A 707, 253 (2002).

11. H. Vonach, R.C. Haight, G. Winkler, Phys. Rev. C 28, 2278 (1983).

12. A. Kumar, S. Kailas, S. Rathi, K. Mahata, Nucl. Phys. A 776, 105 (2006).

13. P. Reimer et al., Phys. Rev. C 65, 014604 (2001).

14. V. Semkova et al., Nucl. Phys. A 730, 255 (2004).

15. M. Avrigeanu, R.A. Forrest, A.J. Koning, F.L. Roman, V. Avrigeanu (this conference).

16. O. Bersillon, Centre d'Études de Bruyères-le-Châtel, Note CEAN-2227, 1992.

17. Dao T. Khoa, W. von Oertzen, Phys. Lett. B 304, 8 (1993); 342, 6 (1995); Dao T. Khoa, W. von Oertzen, H.G. Bohlen, Phys. Rev. C 49, 1652 (1994); Dao T. Khoa, W. von Oertzen, A.A. Ogloblin, Nucl. Phys. A 602, 98 (1996).

18. Experimental Nuclear Reaction Data (EXFOR), http://wwwnds.iaea.or.at/exfor.

19. L. McFadden, G.R. Satchler, Nucl. Phys. A 84, 177 (1966).

20. M. Avrigeanu, V. Avrigeanu, STAPRE-H95 Computer Code, IPNE Report NP-86-1995, Bucharest, 1995; News NEA Data Bank 17, 22 (1995). 